\title{
Foliage Plants Cause Physiological and Psychological Relaxation as Evidenced by Measurements of Prefrontal Cortex Activity and Profile of Mood States
}

\author{
Sin-Ae Park \\ Department of Environmental Health Science, Konkuk University, \\ 05029, Seoul, South Korea
}

\section{Chorong Song}

Center for Environment, Health and Field Sciences, Chiba University, 277-0882, Chiba, Japan

\section{Ji-Young Choi}

Graduate School of Environmental Science, Konkuk University, 05029, Seoul, South Korea

\section{Ki-Cheol Son \\ Department of Environmental Health Science, Konkuk University, 05029, Seoul, South Korea; and the Graduate School of Environmental Science, Konkuk University, 05029, Seoul, South Korea \\ Yoshifumi Miyazaki ${ }^{1}$ \\ Center for Environment, Health and Field Sciences, Chiba University, 277-0882, Chiba, Japan}

Additional index words. brain activity, horticultural therapy, human issues in horticulture, near-infrared spectroscopy, semantic differential method

\begin{abstract}
The study's objective was to investigate the effects of foliage plants on prefrontal cortex activity and subjective assessments of psychological relaxation. In a crossover experimental design, 24 male university students in their 20 s observed a container with and without foliage plants for 3 minutes while oxyhemoglobin (oxy-Hb) concentration in the prefrontal cortex was continuously measured with a portable near-infrared (NIR) spectroscopy device. Afterward, subjective evaluations of emotions were obtained via two self-report questionnaires: a modified semantic differential (SD) method and the Profile of Mood State questionnaire (POMS). Oxy-Hb concentration in the right prefrontal cortex was significantly lower in subjects who viewed the foliage plants than in those who did not, indicating a physiologically relaxed state. The subjects also reported in the SD method significantly more positive emotions (e.g., comfortable, natural, and relaxed) associated with viewing the foliage plants. In the POMS, a significant positive effect on psychological relaxation when subjects viewed the foliage plants was shown. Thus, we conclude that foliage plants have both physiological and psychological relaxation effects in males even after only short exposure.
\end{abstract}

In an urban society, city dwellers spend over $80 \%$ of their time in indoor environments (Yoo and Lee, 2014). Therefore, there are limited opportunities to experience nature or green plants (Lee, 2007). Furthermore, many city dwellers in modern society spend most of their time in a stressed state (Herzog et al., 1997; Kim et al., 2013).

Various theories have been proposed to explain the importance of experiencing nature

Received for publication 27 June 2016. Accepted for publication 14 Aug. 2016.

This paper was supported by the SMART Research Professor Program of Konkuk University.

${ }^{1}$ Corresponding author. E-mail: ymiyazaki@faculty. chiba-u.jp. negative emotions and autonomic nervous system activation. In addition, Ikei et al. (2014a) showed that viewing green plants in an indoor environment was associated with greater stabilization of the autonomic nervous system and parasympathetic activity by measuring heart rate variability. Son et al. (1998) reported that green plants in an interior space increased alpha waves in electroencephalographic activity, which was used as an index of emotional stabilization.

Measurements of brain function are routinely performed using electroencephalography, positron emission tomography, single-photon emission computed tomography, functional magnetic resonance imaging, and magnetoencephalography. However, the experimental environments for these techniques are quite different from normal daily environments, requiring strict motion restriction that may be stressful for the subjects. Furthermore, in vivo determination of the brain activity in humans requires flexible, accessible, and rapid monitoring techniques. Jöbsis (1977) demonstrated that near-infrared spectroscopy (NIRS) can be used as a tool to noninvasively monitor cerebral blood oxygenation. A major advantage of NIRS is better tolerance to head motion because of the temporal resolution of the device (Perrey, 2012) so that NIRS has been used in many studies of activation-induced changes in brain activity over the past 20 years (Perrey, 2012). Near-IR light penetrates tissue to a depth of several centimeters and allows to continuous measure for the noninvasive monitoring of hemodynamics in the prefrontal cortex of the brain (Jöbsis, 1977; Perrey, 2008).

The biological effects of viewing foliage plants on the wellbeing of individuals are not well understood. Because of the advantages of using NIRS to assess brain activity, the objective of this study was to use this technique to investigate the effects of foliage plants on prefrontal cortex activity and associate these effects with subjective assessments of psychological relaxation.

\section{Materials and Methods}

Subjects. University male students in their 20s were recruited from Konkuk University, Seoul, South Korea. Researchers contacted students who were on a campus volunteer list by phone or face to face. In total, 24 male university students were recruited. Inclusion criteria were not having rhinitis symptoms (because these can affect the data) and not consuming caffeine or smoking $2 \mathrm{~h}$ before testing. Researchers provided the study's purpose, procedures, schedule, and requirements to participants, and the subjects provided written informed consent.

The mean age of the subjects in this study was $24.0 \pm 2.7$ years. The mean height and weight were $172.9 \pm 6.4 \mathrm{~cm}$ and $68.6 \pm 11.4 \mathrm{~kg}$, respectively. Body mass index (BMI) was $22.8 \pm 2.9 \mathrm{~kg} \cdot \mathrm{m}^{-2}$, which is in the normal range (WHO, 2006). After completion of the study, the subjects received $\$ 20$ as an 
incentive. The study was approved by the institutional review board (7001355201508-HR-079).

Experimental procedures. A space $(1.5 \times$ $1.7 \mathrm{~m}$ ) with a table and chair in a laboratory at the Konkuk University campus was used in this study. The experimental space in the laboratory was sectioned off by a curtain to avoid possible barrier factors, such as noise or interruption. The space was maintained at (mean $\pm \mathrm{SD}$ ) $25.4 \pm 1.5{ }^{\circ} \mathrm{C}, 52.5 \pm 5.8 \%$ relative humidity, and $1645 \pm 1891 \mathrm{x}$ illumination.

The experimental procedures are presented in Fig. 1. This study was of a crossover experimental design, which is a repeated measures design. In a crossover design, each experimental group crosses over from one treatment to another, which provides a more efficient comparison of treatments than a parallel design (Piantadosi, 2005). Each subject visited the laboratory once to complete this study.

A container with or without plants was covered by a cardboard box on the desk. After a 30-s resting period, each subject randomly observed either foliage plants (Epipremnum aureum $)$ in a container $(55 \times 19 \times 15 \mathrm{~cm})$ or a container without foliage plants for $3 \mathrm{~min}$ while maintaining the sitting position (Fig. 2A and B). Before visual stimulation, the container with or without plants was covered by a cardboard box (Ikei et al., 2014a). During the testing procedure, oxy$\mathrm{Hb}$ concentration in the prefrontal cortex was measured continuously with NIRS as an index of the physiological effects of viewing plants on the prefrontal cortex activity. After finishing the 3-min treatment, subjective evaluations of the emotional effects were measured by using surveys for about $1 \mathrm{~min}$.

Before starting the testing, the subjects were provided with a detailed explanation about the procedures of this study. Body weight and height without shoes of each subject were measured by a body fat analyzer (ioi 353; Jawon Medical, Gyeongsan, South Korea) and an anthropometer (Ok7979; Samhwa, Seoul, South Korea). The BMI was calculated with the data for body weight and height $\left\{\mathrm{BMI}=[\right.$ weight $\left.(\mathrm{kg})] /[\text { height }(\mathrm{m})]^{2}\right\}$.

Measurement for prefrontal cortex activity. Oxy-Hb concentrations in the prefrontal cortex were continuously measured using a portable NIRS (Pocket NIRS Duo; Dynasense, Shizuoka, Japan; Watanabe et al., 2012) while each subject was viewing the containers with or without foliage plants and resting. Moreover, baseline data of oxy- $\mathrm{Hb}$ concentration in the prefrontal cortex in each subject was measured by NIRS by sitting on a chair for $3 \mathrm{~min}$. NIRS was worn on the forehead of each subject (Fig. 3), and the oxy$\mathrm{Hb}$ concentrations in the left and right prefrontal cortex were measured at $1 \mathrm{~Hz}$.

Subjective evaluation. A subjective evaluation was conducted using the modified SD method (Osgood et al., 1957). The SD method uses three pairs of adjectives on 13 scales, including "comfortable-uncomfortable," "natural-artificial," and "relaxed-awakening."

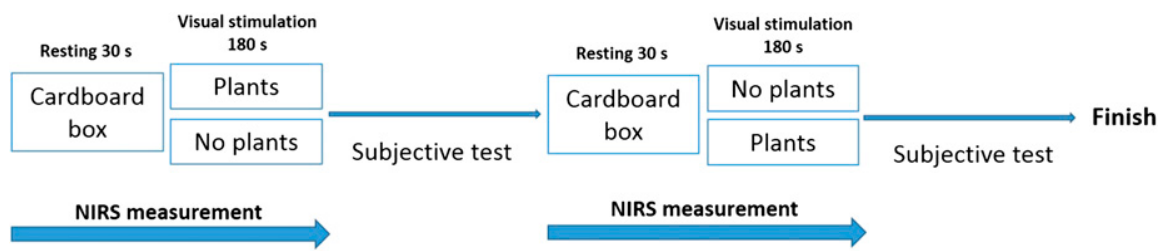

Fig. 1. Study protocol for measuring oxyhemoglobin concentration in the prefrontal cortex. A crossover experimental design is shown for examining the effects of visual stimulation on brain function using near-IR spectroscopy (NIRS) and foliage plants.
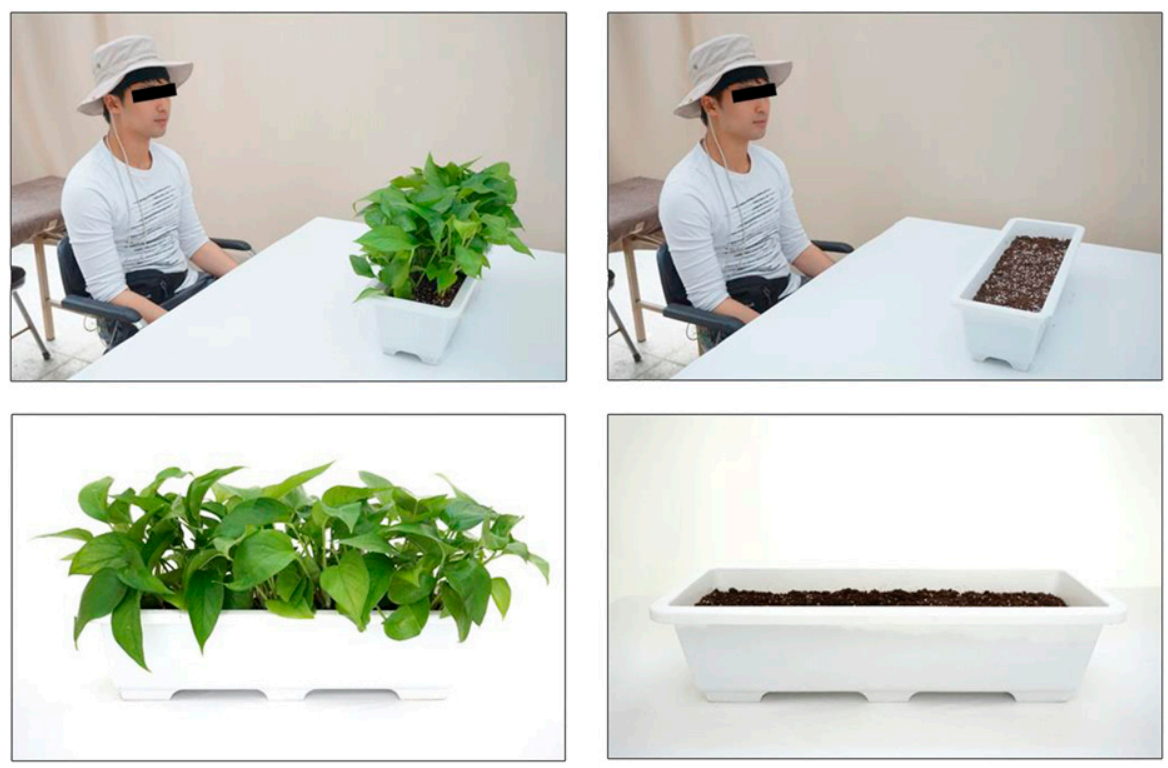

Fig. 2. Experimental viewing conditions: (A) foliage plants (Epiremmun aureum) condition and (B) no foliage plants condition.

A higher score for each indicates a better emotional condition (Osgood, 1952).

In addition, the POMS, originally developed by McNair et al. (2003) and then translated to Korean by Yeun and Shin-Park (2006), was conducted. The POMS questionnaire consists of 30 questions in six subcategories, such as tension-anxiety (T-A), depression-dejection (D), anger-hostility $(\mathrm{A}-\mathrm{H})$, fatigue $(\mathrm{F})$, confusion $(\mathrm{C})$, and vigor (V). The lower scores for the T-A, D, A-H, F, and $\mathrm{C}$ indicate a better emotional condition, but a higher score for the $\mathrm{V}$ indicate a better condition. The total mood disturbance (TMD) score is calculated by the formula $[(\mathrm{T}-\mathrm{A})+$ $(\mathrm{D})+(\mathrm{A}-\mathrm{H})+(\mathrm{F})+(\mathrm{C})-(\mathrm{V})]$ (Baker et al., 2002). A lower score of TMD indicates a better emotional condition. The Chronbach' $\alpha$ was 0.7 (McNair et al., 1992).

Data analysis. Data on the oxy-Hb concentration difference between the control and treatment groups were used for analysis. The measured mean data for the 30 -s resting period served as a baseline measure for each subject. This value was deleted from the oxy-Hb concentration data acquired at every second during the testing period.

Statistical analyses were performed using SPSS software (version 20; SPSS Inc., Chicago, IL) for all variables. $P<0.05$ was considered as statistically significant. The

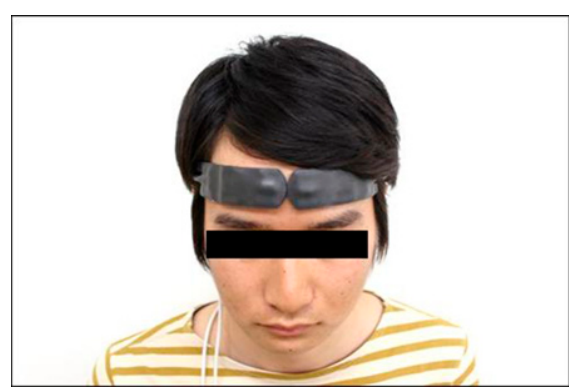

Fig. 3. The portable near-IR spectroscopy (NIRS). Subject wears the NIRS device on the left and right forehead.

differences between the control and treatment groups of oxy-Hb concentration were analyzed using a paired $t$ test. The results from the SD method and POMS were analyzed by the Wilcoxon signed-rank test.

\section{Results}

Prefrontal brain activity measured by NIRS. Changes in oxy-Hb concentration in the right prefrontal cortex over time under the two treatment conditions are shown in Fig. 4. NIRS revealed that the average oxy- $\mathrm{Hb}$ concentration in the right prefrontal cortex in the viewing foliage plant condition was 


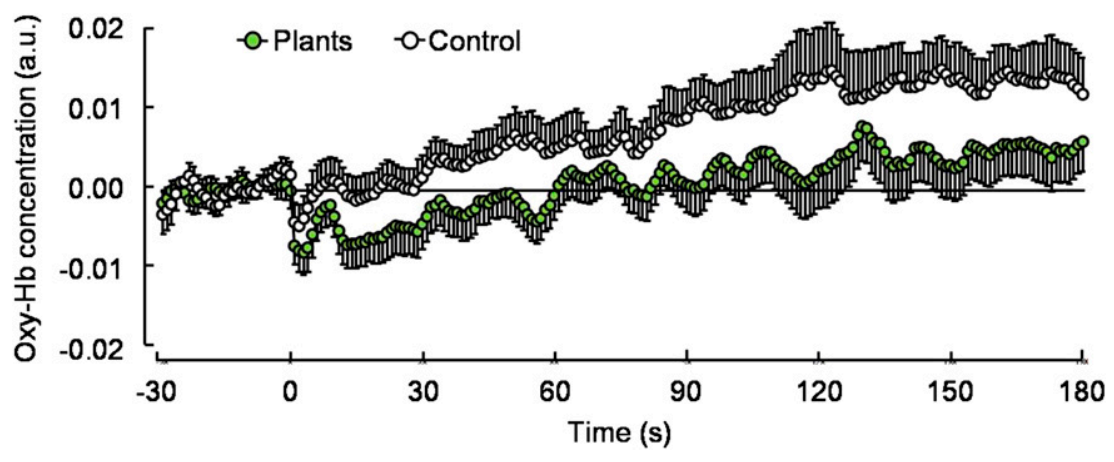

Fig. 4. Changes in oxyhemoglobin (oxy-Hb) concentration in the right prefrontal cortex over time under the two treatment conditions. Values are means $\pm \operatorname{SE}(\mathrm{N}=24)$.

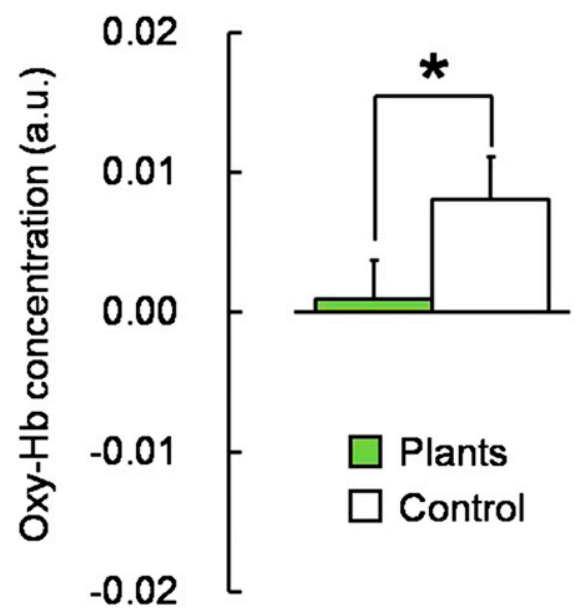

Fig. 5. Comparisons of oxyhemoglobin (oxy-Hb concentration in the right prefrontal cortex. Near-IR spectroscopy was used to assess brain oxy $\mathrm{Hb}$ concentrations between the viewing foliage plants and no foliage plants conditions. Values are means $\pm \mathrm{SE} * P<0.05 ; \mathrm{N}=24$.

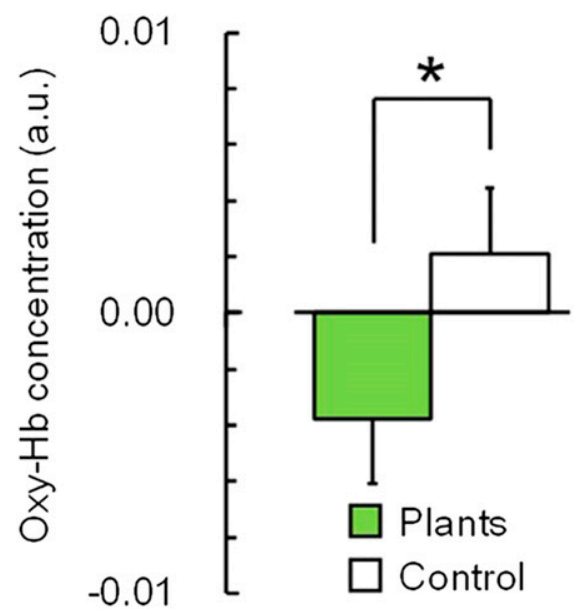

Fig. 6. Comparisons of oxyhemoglobin (oxy-Hb) concentration in the right prefrontal cortex during 1-60 s. Near-IR spectroscopy was used to assess brain oxy $\mathrm{Hb}$ concentrations between the viewing foliage plants and no foliage plants conditions. Values are means $\pm \mathrm{SE}$ $* P<0.05 ; \mathrm{N}=24$.

significantly lower than that in the "no plants" condition for the 1- to 180 -s testing period $(P<0.05$; Fig. 5$)$. At the beginning the observation period of $1-60 \mathrm{~s}$, it was lower than the baseline in the plants group. In the average of 1-60 s, oxy- $\mathrm{Hb}$ concentration in the right prefrontal area was significantly decreased in the viewing foliage plant condition than "no plants" condition $(P<0.05$; Fig. 6). In contrast, there was no significant difference in oxy-Hb concentration in the left prefrontal cortex between the two treatments (data not shown).

Subjective evaluation. The subjective evaluation of the emotional impact of the foliage plants showed an association with positive effects in this study (Figs. 7-9). By the modified SD method, the subjects reported feeling significantly more comfortable, natural, and relaxed when viewing foliage plants than when they were not $(P<$ 0.01; Fig. 7).

The results of the POMS questionnaire also showed a positive effect on psychological relaxation when subjects viewed the foliage plants (Figs. 8 and 9). Specifically, scores in the T-A, A-H, and F subcategories were significantly lower and scores in the $\mathrm{V}$ subcategory were higher when viewing plants than when not viewing plants $(P<$ 0.05 ; Fig. 8). The TMD score was 1.9 in the group with foliage plants and 4.3 in the group with no foliage plants $(P<0.01$; Fig. 9).

\section{Discussion}

The subjects showed physiological and psychological relaxation resulting from visual stimulation comprising the viewing of foliage plants for $180 \mathrm{~s}$. The oxy-Hb concentration in the right prefrontal cortex was significantly lower when viewing the container with, than without, foliage plants, and the decrease started from as early as 1-60 s. Thus, the visual stimulation of viewing foliage plants led to a physiological relaxation effect. In addition, the positive physiological responses of humans to foliage plants appeared almost immediately.

Although few studies have previously used NIRS to examine the effects of visual stimulation on relaxation, a recent study reported a physiological relaxation effect associated with decreasing oxy-Hb concentration in the right prefrontal cortex while viewing a 3-dimensional image of a water lily (Igarashi et al., 2014c). In addition, olfactory stimulation with rose and orange oil induced physiological and psychological relaxation by decreasing the oxy-Hb concentration in the right prefrontal cortex (Igarashi et al., 2014a). Further, Hinoki cypress leaf oil reduced oxy-Hb concentration in the right prefrontal cortex and increased parasympathetic nervous activity (Ikei et al., 2015). The present study led the same results like the previous findings in the physiological and psychological relaxation by decreasing the oxy- $\mathrm{Hb}$ concentration in the right prefrontal cortex. When the local brain activity increases, brain blood flow increases (Fox and Raichle, 1986). The level of brain blood flow is consistent with the level of oxy-Hb (Hoshi et al., 2001), and NIRS can detect this activity-related increase in oxy-Hb. Consequently, it is considered that a decrease in the oxy-Hb concentration equates to a physiological relaxation effect (Igarashi et al., 2014a).

In this study, the left and right prefrontal cortices differentially responded to the viewing of foliage plants. Oxy-Hb concentration measured by NIRS significantly decreased in the right prefrontal area but not in the left prefrontal area. These results are consistent with previous findings: most of the NIRS studies on visual stimulation have reported that only one prefrontal area is significantly activated (Igarashi et al., 2014a, 2014b, 2014c, 2015). Moreover, a review of functional NIRS studies reported hemispheric differences in brain activation (Homae, 2014). The prefrontal region is involved in decision-making and emotional processing (Barrash et al., 2000; Tranel et al., 2000). Although it is unknown whether the right and left prefrontal regions make asymmetric contributions, considerable evidence exists that supports a predominant role for the right hemisphere in emotional processing (Damasio, 1994, 1999), with, in particular, a more dramatic effect on autonomic responses (Angrilli et al., 1999; Zahn et al., 1999). More studies are needed using NIRS to investigate the hemispheric differences in the response to sensory stimulation to clarify the functional implications.

The subjective evaluation of the emotional impact of viewing foliage plants also showed a significant positive effect in this study. The subjects felt comfortable, natural, and relaxed by viewing foliage plants. Furthermore, the subjects reported significantly decreased tension, anxiety, and fatigue and increased vigor. In a previous study, high school students also reported on the SD questionnaire that foliage plants led to positive associations, such as feeling comfortable, relaxed, and natural (Ikei et al., 2014a). Moreover, viewing real pansies compared with artificial pansies produced similar positive emotions in the SD method (Igarashi et al., 2015). Further, via the POMS questionnaire, office workers reported psychological relaxing effects when viewing roses, such as decreased tension, depression, anger, fatigue, and confusion and increased vigor (Ikei et al., 2014b). 


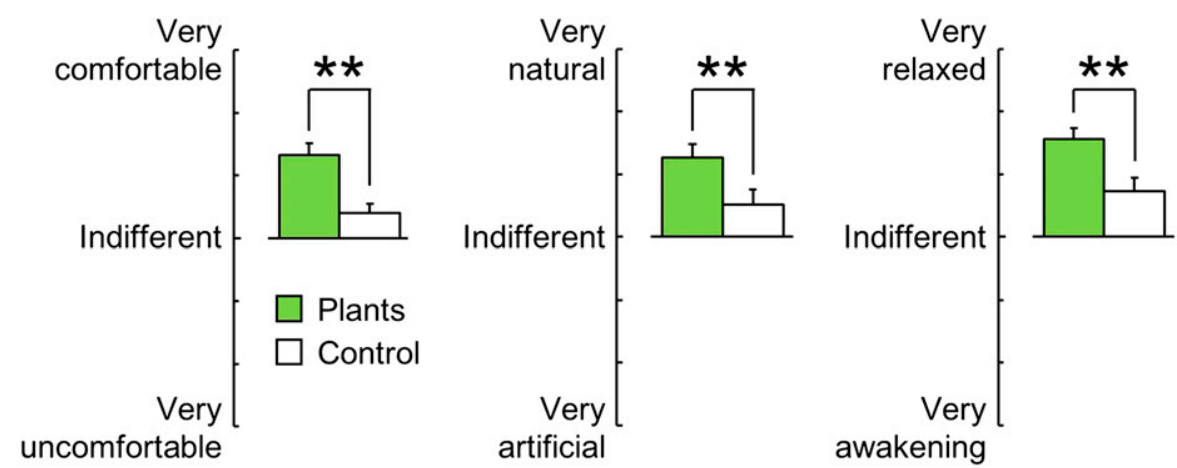

Fig. 7. Comparisons of a modified semantic differential (SD) method under conditions of viewing foliage plants vs. no foliage plants. The SD method uses three pairs of adjectives on 13 scales, including "comfortable-uncomfortable," "natural-artificial," and "relaxed-awakening." A higher score for each indicates better emotional condition. Values are means $\pm \mathrm{SE} * *<0.01 ; \mathrm{N}=24$.

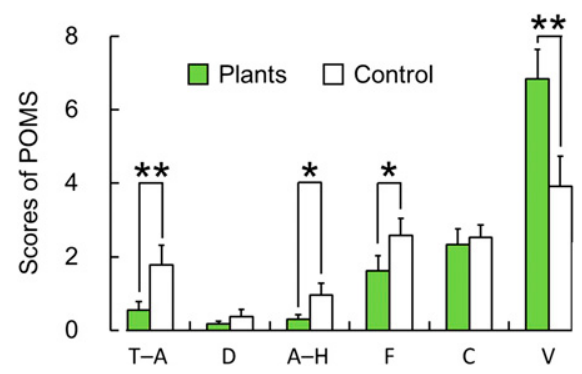

Fig. 8. Comparisons of tension-anxiety (T-A), depression-dejection (D), anger-hostility (A-H), fatigue $(\mathrm{F})$, confusion $(\mathrm{C})$, and vigor $(\mathrm{V})$ in the Profile of Mood State (POMS) questionnaire between conditions of viewing and not viewing foliage plants. The POMS questionnaire consists of 30 questions in the six subcategories of T-A, D, A-H, F, C, and V. Lower scores for T-A, D, A-H, F, and C indicate a better emotional condition, but a higher score for the $\mathrm{V}$ category indicates a better condition. Values are means $\pm \mathrm{SE} * P<0.05$ and $* * P<0.01 ; \mathrm{N}=24$.

\section{$\square$ Plants $\square$ Control}

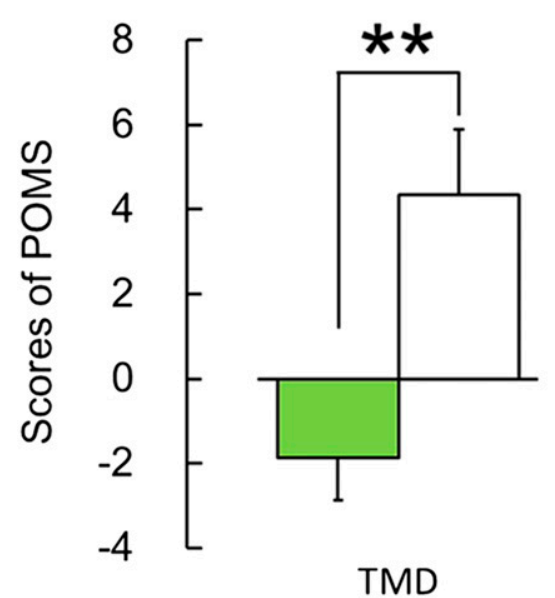

Fig. 9. Comparisons of the total mood disturbance (TMD) score in the Profile of Mood State (POMS) questionnaire between conditions. The POMS questionnaire consists of 30 questions in six subcategories, such as tension-anxiety (T-A), depression-dejection (D), anger-hostility (A-H), fatigue $(\mathrm{F})$, confusion $(\mathrm{C})$, and vigor $(\mathrm{V})$. The TMD score was calculated by the formula $[(\mathrm{T}-\mathrm{A})+(\mathrm{D})+(\mathrm{A}-\mathrm{H})+(\mathrm{F})+(\mathrm{C})-(\mathrm{V})]$. Values are means $\pm \mathrm{SE} * * P<0.01 ; \mathrm{N}=24$.
Previous studies have reported that exposure to environments with plants can have physiological and psychological benefits (Hartig et al., 1991; Ulrich, 1981; Ulrich, 1991; Ulrich and Parsons, 1992). For example, Ulrich (1991) reported that a natural environment led to recovery from stress. In addition, interior spaces with green plants induced stable autonomic nervous system activity (Ikei et al., 2014a) and increased emotional stability as inferred based on increased alpha wave activity in electroencephalographic measurements (Son et al., 1998). Chang and Chen (2005) comprehensively investigated the effects of window views and indoor plants on human psychophysiological responses in workplace environments by measuring electromyography, electroencephalography, blood volume pulse, and state anxiety. The results of this study showed that participants were notably less nervous or anxious when viewing nature or indoor plants. Finally, a review of 21 studies concluded that passive interactions with indoor plants provide psychological benefits, such as stress reduction and increased pain tolerance (Bringslimark et al., 2009).

As our data indicate, even a small number of plants can offer psychophysiological benefits. In accordance with our findings, Ikei et al. (2014a) reported that as few as three foliage plants that were $55-60 \mathrm{~cm}$ in height provided significant physiological and psychological relaxation in high school students. Moreover, Choi (2015) reported that an index of greenness equivalent to $5 \%$ foliage green plants within an interior space resulted in psychophysiological stability in male university students.

In conclusion, in the current study, viewing foliage plants led to physiological and psychological relaxation in male subjects in their 20 s. Future study is needed to investigate the effects on brain function from sensory stimulation for various age groups and gender differences. Furthermore, it would be of interest to determine whether foliage plants could alleviate aggressiveness or emotional instability in individuals with special needs.

\section{Literature Cited}

Angrilli, A., D. Palomba, A. Cantagallo, A. Maietti, and L. Stegagno. 1999. Emotional impairment after right orbitofrontal lesion in a patient without cognitive deficits. Neuroreport 10:1741-1746.

Baker, F., M. Denniston, J. Zabora, A. Polland, and W.N. Dudley. 2002. A POMS short form for cancer patients: Psychometric and structural evaluation. Psychooncology 11:273-281.

Barrash, J., D. Tranel, and S.W. Anderson. 2000. Acquired personality disturbances associated with bilateral damage to the ventromedial prefrontal region. Dev. Neuropsychol. 18:355381.

Bowler, D., L.M. Buyung-Ali, T.M. Knight, and A.S. Pullin. 2010. The importance of nature for health: Is there a specific benefit of contact with green space? Systematic review. CEE review 40:1-57.

Bringslimark, T., T. Hartig, and G.G. Patil. 2009. The psychological benefits of indoor plants: A critical review of the experimental literature. J. Environ. Psychol. 29:422-433.

Chang, C.Y. and P.K. Chen. 2005. Human response to window views and indoor plants in the workplace. HortScience 40:1354-1359.

Choi, J.Y. 2015. Psychophysiological response according to the index of greenness of interior space. MS thesis, Konkuk Univ., Seoul, Korea.

Damasio, A. 1994. Descartes' error: Emotion, reason and the human mind. p. 195-201. Putnam, New York, NY.

Damasio, A.R. 1999. The feeling of what happens: Body and emotion in the making of consciousness. Harcourt Brace, San Diego, CA.

Fox, P.T. and M.E. Raichle. 1986. Focal physiological uncoupling of cerebral blood flow and oxidative metabolism during somatosensory stimulation in human subjects. Proc. Natl. Acad. Sci. 83:1140-1144.

Hartig, T., M. Mang, and G.W. Evans. 1991. Restorative effects of natural environment experiences. Environ. Behav. 23:3-26.

Herzog, T.R., A.M. Black, K.A. Fountaine, and D.J. Knotts. 1997. Reflection and attentional recovery as distinctive benefits of restorative environments. J. Environ. Psychol. 17:165-170.

Homae, F. 2014. A brain of two halves: Insights into interhemispheric organization provided by near-infrared spectroscopy. Neuroimage 85:354-362.

Hoshi, Y., N. Kobayashi, and M. Tamura. 2001. Interpretation of near-infrared spectroscopy signals: A study with a newly developed perfused rat brain model. J. Appl. Physiol. 90:1657-1662.

Igarashi, M., M. Aga, H. Ikei, T. Namekawa, and Y. Miyazaki. 2015. Physiological and psychological effects on high school students of viewing real and artificial pansies. Int. J. Environ. Res. Public Health. 12:2521-2531.

Igarashi, M., H. Ikei, C. Song, and Y. Miyazaki. 2014a. Effects of olfactory stimulation with rose and orange oil on prefrontal cortex activity. Complement. Ther. Med. 22:10271031.

Igarashi, M., C. Song, H. Ikei, and Y. Miyazaki. 2014b. Effects of olfactory stimulation with perilla essential oil on prefrontal cortex activity. J. Altern. Complement. Med. 20:545-549.

Igarashi, M., T. Yamamoto, J. Lee, C. Song, H. Ikei, and Y. Miyazaki. 2014c. Effects of stimulation by three-dimensional natural images on prefrontal cortex and autonomic nerve activity: A comparison with stimulation using twodimensional images. Cogn. Process. 15:551556. 
Ikei, H., C. Song, and Y. Miyazaki. 2015. Physiological effect of olfactory stimulation by Hinoki cypress (Chamaecyparis obtusa) leaf oil. J. Physiol. Anthropol. 34:44.

Ikei, H., C. Song, M. Igarashi, T. Namekawa, and Y. Miyazaki. 2014a. Physiological and psychological relaxing effects of visual stimulation with foliage plants in high school students. Adv. Hort. Sci. 28:111-116.

Ikei, H., M. Komatsu, C. Song, E. Himoro, and Y. Miyazaki. 2014b. The physiological and psychological relaxing effects of viewing rose flowers in office workers. J. Physiol. Anthropol. 33:6.

Jöbsis, F.F. 1977. Noninvasive, infrared monitoring of cerebral and myocardial oxygen sufficiency and circulatory parameters. Science 198:1264-1267.

Kaplan, R. and S. Kaplan. 1989. The experience of nature: A psychological perspective. Cambridge University Press, Cambridge, UK.

Kim, J.H., S.Y. Sun, and Y.H. Yoon. 2013. The effects of urban stream landscape on psychological relaxation of university students: Focused on Chenggyecheon, Seoul, Korea. Seoul Studies 14:169-182.

Lee, S.H. 2007. A comparison of the models for explaining the emotion-improving effects of the index of greenness. Kor. J. Health Psychology 12:189-217.

McNair, D.M., J.P. Heuchert, and E. Shilony. 2003. Profile of mood states bibliography 1964-2002. Multi-Health Systems Inc., North Tonawanda, NY.

McNair, D.M., M. Lorr, and L.F. Droppleman. 1992. Manual for the profile of mood states.
Educational and Industrial Testing Service, San Diego, CA.

Osgood, C.E. 1952. The nature and measurement of meaning. Psychol. Bull. 49:197-237.

Osgood, C.E., G.J. Suci, and P.H. Tannenbaum. 1957. The measurement of meaning. University of Illinois Press, Chicago, IL.

Perrey, S. 2008. Non-invasive NIR spectroscopy of human brain function during exercise. Methods 45:289-299.

Perrey, S. 2012. NIRS for measuring cerebral hemodynamic responses during exercise, $\mathrm{p}$. 335-349. In: H. Boecker, C.H. Hillman, L. Scheef, and H.K. Strüder (eds.). Functional neuroimaging in exercise and sport sciences. Springer-Verlag, New York, NY.

Piantadosi, S. 2005. Crossover designs, p. 515-527. In: P. Steven (ed.). Clinical Trials: A methodologic perspective. Wiley, New York, NY.

Son, K.C., J.S. Lee, and J.E. Song. 1998. Effect of visual recognition of indoor plants on changes of human brain electroencephalography. J. Kor. Sor. Hort. Sci. 39:858-862.

Tranel, D., A. Bechara, and A.R. Damasio. 2000. Decision making and the somatic marker hypothesis, p. 1047-1061. In: M.S. Gazzaniga (ed.). The new cognitive neurosciences. The MIT Press, Cambridge, MA.

Ulrich, R.S. 1981. Natural versus urban scenes some psychophysiological effects. Environ. Behav. 13:523-556.

Ulrich, R.S. 1983. Aesthetic and affective response to natural environment, p. 85-125. In: I. Altman and J.F. Wohlwill (eds.). Behavior and the natural environment. Springer US, Plenum Press, New York, NY.

Ulrich, R.S. 1991. Psychophysiological indicators of leisure, p. 73-89. In: B.L. Driver, P.J. Brown, and G.L. Peterson (eds.). Benefits of leisure. Venture Publishing Inc, State College, PA.

Ulrich, R.S. and R. Parsons. 1992. Influences of passive experiences with plants on individual well-being and health, p. 93-105. In: D. Relf (ed.). The role of horticulture in human wellbeing and social development. Timber press, Portland, OR.

Watanabe, T., T. Mizuno, T. Shikayama, and M. Miwa. 2012. Development of a wireless nearinfrared tissue oxygen monitor system with high sampling rate. In Biomedical Optics (pp. JM3A-11). Optical Soc. Amer.

World Health Organization (WHO). 2006. BMI classification. Global database on body mass index. 27 July 2012. apps.who.int/bmi/index. jsp.

Yeun, E.J. and K.K. Shin-Park. 2006. Verification of the profile of mood states-brief: Cross-cultural analysis. J. Clin. Psychol. 62:1173-1180.

Yoo, M. and E.H. Lee. 2014. The impact of modulized interior landscape on office workers' psychological wellbeing-A pilot study of focused on the office wall. Kor. Institute Interior Design J. 23:220-239.

Zahn, T.P., J. Grafman, and D. Tranel. 1999. Frontal lobe lesions and electrodermal activity: Effects of significance. Neuropsychologia 37:1227-1241. 